\title{
Circ-FBXW12 aggravates the development of diabetic nephropathy by binding to miR-31-5p to induce LIN28B
}

\author{
Aidong Sun ${ }^{1}$, Ningshuang Sun ${ }^{2}$, Xiao Liang ${ }^{3}$ and Zhenbo Hou ${ }^{4 *}$
}

\begin{abstract}
Background: The involvement of circular RNAs (circRNAs) in diabetic nephropathy (DN) has been gradually identified. In this study, we aimed to explore the functions of circRNA F-box/WD repeat-containing protein 12 (circ-FBXW12) in DN development.

Methods: Reverse transcription quantitative polymerase chain reaction (RT-qPCR) assay was performed for the levels of circ-FBXW12, FBXW12 mRNA, microRNA-31-5p (miR-31-5p) and Lin-28 homolog B (LIN28B) mRNA. RNase R assay was used to analyze the stability of circ-FBXW12. Cell Counting Kit-8 (CCK-8) assay, flow cytometry analysis and 5-ethynyl-2'- deoxyuridine (EdU) assay were employed to evaluate cell viability, cell cycle and proliferation, respectively. Enzyme linked immunosorbent assay (ELISA) was done to measure the concentrations of inflammatory cytokines. Western blot assay was conducted for protein levels. Superoxide dismutase (SOD) activity and malondialdehyde (MDA) level were examined with commercial kits. Dual-luciferase reporter assay and RNA immunoprecipitation (RIP) assay were performed to verify the relationships among circ-FBXW12, miR-31-5p and LIN28B.

Results: Circ-FBXW12 level was increased in DN patients' serums and high glucose (HG)-induced human mesangial cells (HMCs). Circ-FBXW12 knockdown suppressed cell proliferation, arrested cell cycle, reduced extracellular matrix (ECM) production and oxidative stress in HG-induced HMCs. Circ-FBXW12 was identified as the sponge for miR-31-5p, which then directly targeted LIN28B. MiR-31-5p inhibition reversed circ-FBXW12 knockdown-mediated effects on cell proliferation, cell cycle process, ECM production and oxidative in HG-triggered HMCs. Moreover, miR-31-5p overexpression showed similar results with circ-FBXW12 knockdown in HG-stimulated HMC progression, while LIN28B elevation reversed the effects.
\end{abstract}

Conclusion: Circ-FBXW12 knockdown suppressed HG-induced HMC growth, inflammation, ECM accumulation and oxidative stress by regulating miR-31-5p/LIN28B axis.

Keywords: DN, HMCs, Circ-FBXW12, miR-31-5p, LIN28B

*Correspondence: h18678187069@126.com

${ }^{4}$ Department of Pathology, Zibo Central Hospital, No. 54 Gongqingtuan West Road, Zhangdian District, Zibo 255000, Shandong, People's Republic of China

Full list of author information is available at the end of the article

\section{Introduction}

Diabetes mellitus is a common metabolic disease that can cause chronic renal impairment and lead to diabetic nephropathy (DN) $[1,2]$. At present, DN has been one of the major reasons for end-stage renal disease around the world [3]. The clinical features of DN include mesangial cell (MC) hyperplasia, proteinuria, extracellular matrix (ECM) accumulation, and renal fibrosis [4, 5]. Oxidative stress and inflammation caused by elevated blood original author(s) and the source, provide a link to the Creative Commons licence, and indicate if changes were made. The images or other third party material in this article are included in the article's Creative Commons licence, unless indicated otherwise in a credit line to the material. If material is not included in the article's Creative Commons licence and your intended use is not permitted by statutory regulation or exceeds the permitted use, you will need to obtain permission directly from the copyright holder. To view a copy of this licence, visit http://creativecommons.org/licenses/by/4.0/. The Creative Commons Public Domain Dedication waiver (http://creativecommons.org/publicdomain/zero/1.0/) applies to the data made available in this article, unless otherwise stated in a credit line to the data. 
glucose are considered to be inseparable factors in the occurrence of DN [6]. Hence, it is crucial to explore the mechanism of MC injury under high glucose (HG) for understanding DN development.

Circular RNAs (circRNAs) are endogenous non-coding RNAs (ncRNAs) that possess continuous covalent closed loops [7]. Mounting evidence has demonstrated that circRNAs function as vital masters in a variety of diseases by elevating mRNA expression through acting microRNA (miRNA) sponges [8]. Some circRNAs have been implied to be dysregulated and play essential roles in DN pathogenesis. For example, circ_0080425 was upregulated in DN mice model and HG-treated MCs, and promoted MC cell growth and fibrosis via circ_0080425/ miR-24-3p/FGF11 regulatory axis [9]. Circ_0000285 aggravated podocyte proliferation and facilitated apoptosis in DN depending on miR-654-3p/Mitogen-Activated Protein Kinase 6 (MAPK6) axis [10]. Circ_LARP4 hampered cell proliferation and fibrosis and accelerated apoptosis in HG-induced mouse mesangial cells (MMCs) by decoying miR-424 [11]. For circRNA F-box/WD repeatcontaining protein 12 (circ-FBXW12, also termed as hsa circ_0123996), Wang et al. declared that circ_0123996 knockdown suppressed MMC proliferation and fibrosis by upregulating BTB Domain And CNC Homolog 1 (Bach1) via sponging miR-149-5p [12]. Even so, the underlying mechanism of circ-FBXW12 in DN development are poorly characterized.

The small ncRNAs, miRNAs have been demonstrated to be implicated in DN pathogenesis [13]. For example, miR-325-3p restrained renal inflammatory response and fibrosis by interacting with $\mathrm{C}-\mathrm{C}$ motif chemokine ligand 19 (CCL19) [14]. MiR-15b-5p alleviated HG-induced inflammatory damage and oxidative damage in podocytes by binding to semaphorin 3A (Sema3A) [15]. Moreover, miR-31 level was declined in T2D patients with DN and negatively related to the secretion of inflammatory factors [16].
Lin-28 homolog B (LIN28B) is related to multiple human diseases, such as hepatocellular carcinoma [17], neuroendocrine prostate cancer [18], neuroblastoma [19] and Ewing sarcoma [20]. Moreover, LIN28B/let-7 was able to alter ECM production in TGF- $\beta$-induced MMCs [21]. The reports suggested that LIN28B played a vital function in DN.

With the assistance of bioinformatics tools circinteractome and starBase V2.0, miR-31-5p was found to contain the binding sequences of circ-FBXW12 and LIN28B, thus, we explored their functions and relationships in regulating DN development.

\section{Materials and methods}

\section{Clinical sample acquisition}

A total of 23 healthy volunteers who underwent routine health checks (Normal group), 23 type-2 diabetes patients with DN (DN group) and 14 diabetic patients without DN (DM group) at Zibo First Hospital were enrolled in the study. The diabetic patients were diagnosed according to urinary albumin excretion and divided into two groups: diabetes with normoalbuminuria (DM group) (urinary albumin excretion rate (UAER) $<30 \mathrm{mg} / 24 \mathrm{~h}$ and serum creatinine $(\mathrm{Scr})<133 \mu \mathrm{mol} / \mathrm{L})$, diabetes with albuminuria (DN group) (UAER $>30 \mathrm{mg} / 24 \mathrm{~h}$ ). The DN patients were then subdivided into two groups: microalbuminuria group $(30 \mathrm{mg} / 24 \mathrm{~h}<\mathrm{UAER}<300 \mathrm{mg} / 24 \mathrm{~h}$ and macroalbuminuria group (UAER $>300 \mathrm{mg} / 24 \mathrm{~h}$ ). The patients were excluded this study if they had a history of cardiovascular disease, morbified obesity, organic or inflammatory disease, infectious, autoimmune, hematologic disease, malignancy, fever and diabetic neuropathy. The blood samples were acquired after the research was approved by the Ethics Committee of Zibo First Hospital and written informed consents were provided by the participants. The serums were acquired through centrifugation. The clinical characteristics of the participants were exhibited in Table 1.

Table 1 Clinical characteristics

\begin{tabular}{lll}
\hline Parameters & Normal group $(\mathbf{n}=\mathbf{2 3})$ & DN group $(\mathbf{n}=\mathbf{2 3})$ \\
\hline Gender (male/female) & $14 / 9$ & $12 / 11$ \\
Age (years) & $55.3 \pm 5.8$ & $57.2 \pm 6.5$ \\
BMI & $22.6 \pm 1.6$ & $24.8 \pm 2.4$ \\
Duration of diabetes (years) & & $7.9 \pm 1.4$ \\
Fasting plasma glucose (mmol/L) & $4.1 \pm 0.9$ & $7.5 \pm 1.9$ \\
Blood urea nitrogen (mmol/L) & $3.9 \pm 1.2$ & $7.8 \pm 2.8$ \\
HbA1c (mmol/mol) & $74.5 \pm 10.5$ & $40.1 \pm 9.3$ \\
Serum creatinine $(\mu \mathrm{mol} / \mathrm{L})$ & $65.3 \pm 7.6$ & $143.6 \pm 39.2$ \\
Retinopathy & 0 & 11 \\
\hline
\end{tabular}




\section{Cell culture}

HMCs were acquired from Procell (Wuhan, China) and cultured in Dulbecco's modified Eagle's medium (DMEM; Procell) plus 10\% fetal bovine serum (FBS; Procell) and $1 \%$ Penicillin-Streptomycin (Procell) at $37{ }^{\circ} \mathrm{C}$ in a humid incubator consisting of $5 \% \mathrm{CO}_{2}$. The HMCs were treated with $5.5 \mathrm{mM}$ glucose (Sigma-Aldrich, St. Louis, MO, USA) for the control group or $30 \mathrm{mM}$ glucose (Sigma-Aldrich) for the HG group.

\section{Cell transfection}

To knock down circ-FBXW12 or LIN28B, small interfering RNA (siRNA) target circ-FBXW12 (si-circ-FBXW12) or LIN28B (si-LLIN28B) was transfected into HMCs with scramble control si-NC as a control. The mimics of miR-31-5p (miR-31-5p) and inhibitors of miR-31-5p (anti-miR-31-5p) were synthesized to elevate or reduce miR-31-5p expression with miR-NC or anti-miR-NC as the control. To upregulate LIN28B expression, the overexpression plasmid of LIN28B was introduced into HMCs with pcDNA as a control. All these compositions were bought from GenePharma (Shanghai, China) and cell transfection was manipulated utilizing Lipofectamine 2000 (Invitrogen, Carlsbad, CA, USA).

\section{Reverse transcription quantitative polymerase chain reaction (RT-qPCR) assay}

The RNA was obtained utilizing TRIzol (Invitrogen) and cDNAs was generated via All-in-One ${ }^{\mathrm{TM}}$ miRNA FirstStrand cDNA Synthesis Kit (GeneCopoeia, Rockville, MD, USA) or PrimeScript ${ }^{\mathrm{TM}}$ RT reagent Kit (Takara, Dalian, China) according to the manufacturers' instructions. Then RT-qPCR was done via SYBR Premix DimerEraser (Takara). The thermocycling conditions were as follows: (1) $95{ }^{\circ} \mathrm{C}$ for $5 \mathrm{~min}$; (2) 40 cycles at $95{ }^{\circ} \mathrm{C}$ for $30 \mathrm{~s}, 60{ }^{\circ} \mathrm{C}$ for $45 \mathrm{~s}$ and $72{ }^{\circ} \mathrm{C}$ for $30 \mathrm{~s}$; and (3) dissolving curve at $94{ }^{\circ} \mathrm{C}$ for $90 \mathrm{~s}, 60{ }^{\circ} \mathrm{C}$ for $180 \mathrm{~s}$ and $94{ }^{\circ} \mathrm{C}$ for $10 \mathrm{~s}$. The primers were exhibited in Table 2 . The abundance was estimated with the $2^{-\Delta \Delta \mathrm{Ct}}$ method. Glyceraldehyde 3-phosphate dehydrogenase (GAPDH) was used for the internal reference for circ-FBXW12, FBXW12 and LIN28B, while U6 was used for the internal reference for miR-31-5p.

For RNase R assay, total RNA was exposed to RNase R (Epicentre, Madison, WI, USA) for $15 \mathrm{~min}$ at $37^{\circ} \mathrm{C}$. Then the expression levels of circ-FBXW12 and FBXW12 were quantified by RT-qPCR assay.

\section{Subcellular fraction assay}

The PARIS Kit (Invitrogen) was adopted to separate the cytoplasm and nucleus in HMCs according to the manufacturers' instructions. The level of circ-FBXW12 in the cytoplasm and nucleus was quantified.

\section{Cell counting kit-8 (CCK-8) assay}

To test cell viability, CCK-8 assay kit (Sigma-Aldrich) was used. In short, the transfected HMCs were plated into 96-well plates and then CCK- 8 was supplemented into each well for further $2 \mathrm{~h}$ of incubation. The absorption was measured at $450 \mathrm{~nm}$ via a microplate reader (BioRad, Hercules, CA, USA).

\section{Enzyme linked immunosorbent assay (ELISA)}

The concentrations of interleukin- 6 (IL-6) and tumour necrosis factor $\alpha$ (TNF- $\alpha)$ in the culture medium of HMCs were examined by using relevant ELISA kits (ab178013; ab181421; Abcam, Cambridge, MA, USA) referring to the manufacturers' instructions.

Table 2 Primers sequences used for qRT-PCR

\begin{tabular}{|c|c|c|c|c|}
\hline Primers & Sequences $\left(5^{\prime}-3^{\prime}\right)$ & $\mathrm{Tm}$ & PCR product & Ct range \\
\hline circ-FBXW12-forward & ACACGTGGCATGATCACACA & 60.25 & 125 & 0.8 \\
\hline circ-FBXW12-reverse & ACTCCTGGACTGGGCTTGAC & 58.4 & & \\
\hline FBXW12-forward & CTGCCTGGGTTAAGAGATGTTT & 58.31 & 115 & 1.2 \\
\hline FBXW12-reverse & AGGTACGACTGTATGTCCCAC & 58.9 & & \\
\hline miR-31-5p-forward & ACACTCCAGCTGGGAGGCAAGATGCTGGC & 62.43 & 69 & 1.7 \\
\hline miR-31-5p-reverse & TGGTGTCGTGGAGTCG & 55.46 & & \\
\hline LIN28B-forward & TTGAGTCAATACGGGTAACAGGA & 59.17 & 184 & 0.9 \\
\hline LIN28B-reverse & TGACAGTAATGGCACTTCTTTGG & 59.18 & & \\
\hline GAPDH-forward & ACAGTCAGCCGCATCTTCTT & 59.68 & 94 & 1.8 \\
\hline GAPDH-reverse & ACGACCAAATCCGTTGACTC & 58.21 & & \\
\hline U6-forward & CCTCGCTTCGGCAGCACATA & 62.91 & 94 & 1.3 \\
\hline U6-reverse & ACGCTTCACGAATTTGCGT & 59.06 & & \\
\hline
\end{tabular}




\section{Flow cytometry analysis}

To assess cell cycle process, HMCs with relevant transfection were collected, rinsed with phosphate buffer saline (PBS; Sigma-Aldrich) and fixed for $8 \mathrm{~h}$ with $75 \%$ ethanol at $4{ }^{\circ} \mathrm{C}$. Then the cells were resuspended in PBS (Sigma-Aldrich) and stained with propidium iodide (PI; Beyotime, Shanghai, China) supplemented with RNase A (Sigma-Aldrich) for $15 \mathrm{~min}$ in darkness. Cell proportion in each stage was analyzed through FACScan ${ }^{\circledR}$ flow cytometry (BD Biosciences, San Jose, CA, USA).

\section{5-ethynyl-2'-deoxyuridine (EdU) assay}

Cell proliferation was assessed by EdU incorporation kit (RiboBio, Guangzhou, China). In short, HMCs were seeded into 24-well plates and EdU was added into the plates for $2 \mathrm{~h}$. Next, the cells were fixed using $4 \%$ paraformaldehyde (Sigma-Aldrich) for $0.5 \mathrm{~h}$, permeabilized for 10 min using $0.3 \%$ Triton X-100, and rinsed in PBS. Thereafter, the cells were incubated with Aollo fluorescent staining solution for $0.5 \mathrm{~h}$ in darkness. Next, the cells were dyed with Hoechst 33342 solution. The positive cells were counted under a fluorescence microscope (Olympus, Tokyo, Japan).

\section{Western blot assay}

The protein was extracted utilizing RIPA buffer (SigmaAldrich). Equal amount of proteins was subjected to sodium dodecyl sulfonate-polyacrylamide gel (SDSPAGE; Sigma-Aldrich) electrophoresis and then blotted on PVDF membranes (Millipore, Billerica, MA, USA). The membranes were then blocked in skim milk, maintained with primary antibodies CyclinD1 (ab226977; Abcam), P21 (ab109520; Abcam), collagen I (ab34710; Abcam), collagen IV (ab6586; Abcam), TGF$\beta 1$ (ab215715; Abcam), LIN28B (ab115698; Abcam) and GAPDH (ab37168; Abcam) and secondary antibody (ab205719; Abcam). The immunoblotting signals were visualized using an enhanced chemiluminescence (ECL) kit (Beyotime).

\section{Measurement of superoxide dismutase (SOD) activity and malondialdehyde (MDA) level}

The activity of SOD and the content of MDA in HMCs were examined with SOD assay kit (Sigma-Aldrich) and MDA assay kit (Sigma-Aldrich) strictly according to the guidelines.

\section{Dual-luciferase reporter assay}

The wild-type (including miR-31-5p binding sites) or mutant (miR-31-5p binding sites mutation) fragments of circ-FBXW12 or LIN28B 3'UTR were administrated into psiCHECK-2 plasmid (Promega, Madison, WI, USA). Then HMCs were transfected with the constructed vectors and $\mathrm{miR}-31-5 \mathrm{p} / \mathrm{miR}-\mathrm{NC}$. The luciferase intensity was measured with Dual-Luciferase Reporter Assay Kit (Promega) following $48 \mathrm{~h}$ of co-transfection.

\section{RNA immunoprecipitation (RIP) assay}

HMCs were lysed in RIP buffer and cell extracts were cultivated with magnetic beads conjugated with IgG (Abcam) or Ago2 (Abcam). Thereafter, the samples were maintained with proteinase K (Sigma-Aldrich) for $0.5 \mathrm{~h}$ to separate the RNA-protein complexes from beads followed by RT-qPCR assay for the abundance of circFBXW12, miR-31-5p and LIN28B.

\section{Statistical analysis}

The sample size was evaluated by G*Power. The experiments were performed in triple times and the data were estimated by GraphPad Prism 7 and exhibited as mean $\pm S D$. The data were normally distributed. The differences of two sets and three sets were analyzed by Student's $t$-test or one-way analysis of variance followed by Tukey's test. It was considered as significant when $P<0.05$.

\section{Results}

Circ-FBXW12 was highly expressed in DN patients and HG-induced HMCs

To explore the function of circ-FBXW12 in DN progression, the expression of circ-FBXW12 in the serums of DN patients, diabetic patients without DN (DM group) and healthy volunteers was determined by RT-qPCR assay. The results showed that circ-FBXW12 level was apparently increased in DN patients and DM patients in comparison with normal controls (Fig. 1A). Moreover, circ-FBXW12 was markedly increased in HG-treated HMCs compared to control groups (Fig. 1B). RNase $\mathrm{R}$ assay indicated that circ-FBXW12 was resistant to RNase R treatment, while linear FBXW12 was digested by RNase $\mathrm{R}$ treatment (Fig. 1C). Moreover, our results exhibited that circ-FBXW12 was mainly enriched in the cytoplasm of HMCs (Fig. 1D). These results suggested the potential role of circ-FBXW12 in DN development.

\section{Silencing of circ-FBXW12 suppressed HG-induced cell proliferation, inflammation, cell cycle, ECM production and oxidative stress in HMCs}

To explore the exact roles of circ-FBXW12 in DN development, HMGs were transfected with si-circ-FBXW12 to knock down circ-FBXW12 expression in HMGs. As a result, the upregulation of circ-FBXW12 in HMGs caused by HG treatment was reversed by the transfection of si-circFBXW12 (Fig. 2A). CCK-8 assay indicated that HG treatment led to a distinct promotion in the viability of HMCs compared to control group, while circ-FBXW12 

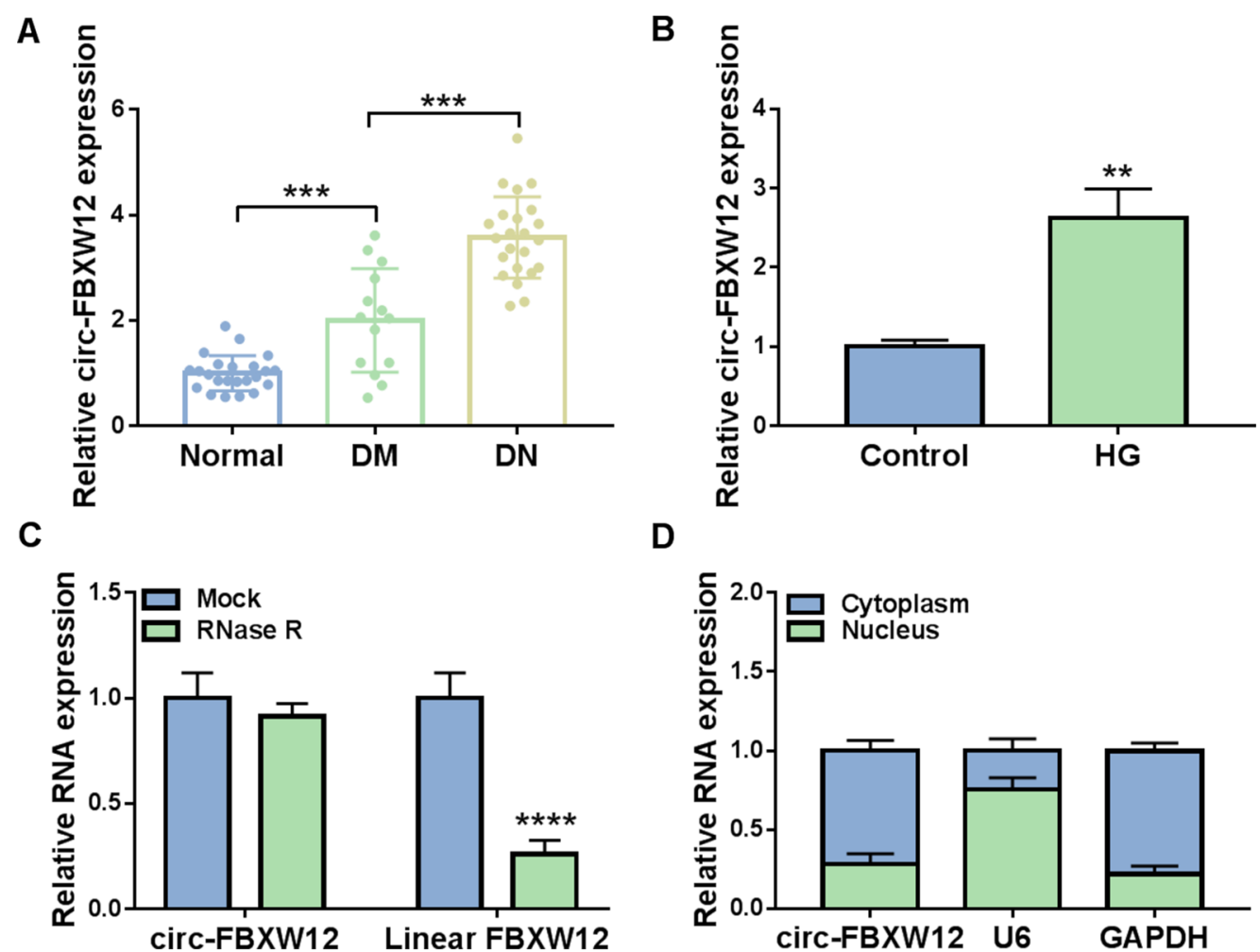

D

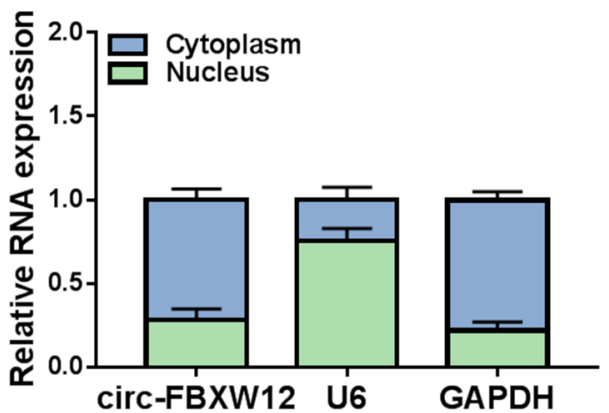

Fig. 1 Circ-FBXW12 was increased in DN patients and HG-treated HMCs. A The expression of circ-FBXW12 in the serums of DN patients, diabetic patients without DN and normal controls was detected by RT-qPCR assay. B The expression of circ-FBXW12 in HG-stimulated HMCs was detected by RT-qPCR assay. C The levels of circ-FBXW12 and FBXW12 in HMCs treated with or without RNase R were determined using RT-qPCR assay. D The expression of circ-FBXW12 in the cytoplasm and nucleus of HMCs was examined by RT-qPCR assay. ${ }^{* *} P<0.01,{ }^{* * *} P<0.0001$

silencing reversed the effect (Fig. 2B). ELISA showed that the concentrations of IL- 6 and TNF- $\alpha$ were increased in HMCs treated with HG, whereas the effects were abated by downregulating circ-FBXW12 (Fig. 2C). As illustrated by flow cytometry analysis, the percentage of HMCs in G0/G1 phases was reduced and the percentage of HMCs in $\mathrm{S}$ phase was increased after HG exposure, while circFBXW12 knockdown ameliorated the effects (Fig. 2D). EdU assay indicated that HG treatment promoted HMC proliferation, with circ-FBXW12 deficiency rescued the impact (Fig. 2E). HG treatment increased CyclinD1 protein level and decreased P21 level in HMCs, with circ-FBXW12 silencing rescued the effects (Fig. 2F). Moreover, we found that HG treatment increased the protein levels of ECM markers (collagen I and collagen IV) in HMCs, while the effects were overturned by decreasing circ-FBXW12 (Fig. 2G). TGF- $\beta 1$ is closely related to the production of ECM [22]. Thus, we detected the protein level of TGF- $\beta 1$ in HG-treated HMCs. Our results showed that TGF- $\beta 1$ was elevated in HG-treated HMCs, but circ-FBXW12 interference reversed the effect (Fig. 2H). Besides, it was found that the activity of SOD was inhibited and the level of MDA was increased in
HG-treated HMCs, while circ-FBXW12 silencing rescued the effects (Fig. 2I and J). Taken together, HG treatment promoted cell proliferation, inflammation, cell cycle process, ECM production and oxidative stress in HMCs, with circFBXW12 silencing abrogated the impacts.

\section{Circ-FBXW12 acted as miR-31-5p sponge}

By using bioinformatics prediction software circinteractome (https://circinteractome.irp.nia.nih.gov/), miR-31-5p was found to share the binding sites of circ-FBXW12 (Fig. 3A). As exhibited in Fig. 3B, the transfection of miR31-5p led to a marked elevation in miR-31-5p expression in HMCs. Dual-luciferase reporter assay showed that miR31-5p overexpression dramatically inhibited the luciferase activity of WT-circ-FBXW12 in HMCs, but did not affect the luciferase activity of MUT-circ-FBXW12 (Fig. 3C). RIP assay showed that the levels of miR-31-5p and circFBXW12 were notably enriched in Ago2 RIP groups compared to IgG groups (Fig. 3D). These results indicated the interaction between miR-31-5p and circ-FBXW12. Indeed, miR-31-5p was weakly expressed in $\mathrm{DN}$ patients, DM patients and HG-treated HMCs compared to control groups (Fig. 3E and F). As analyzed by Spearman's 


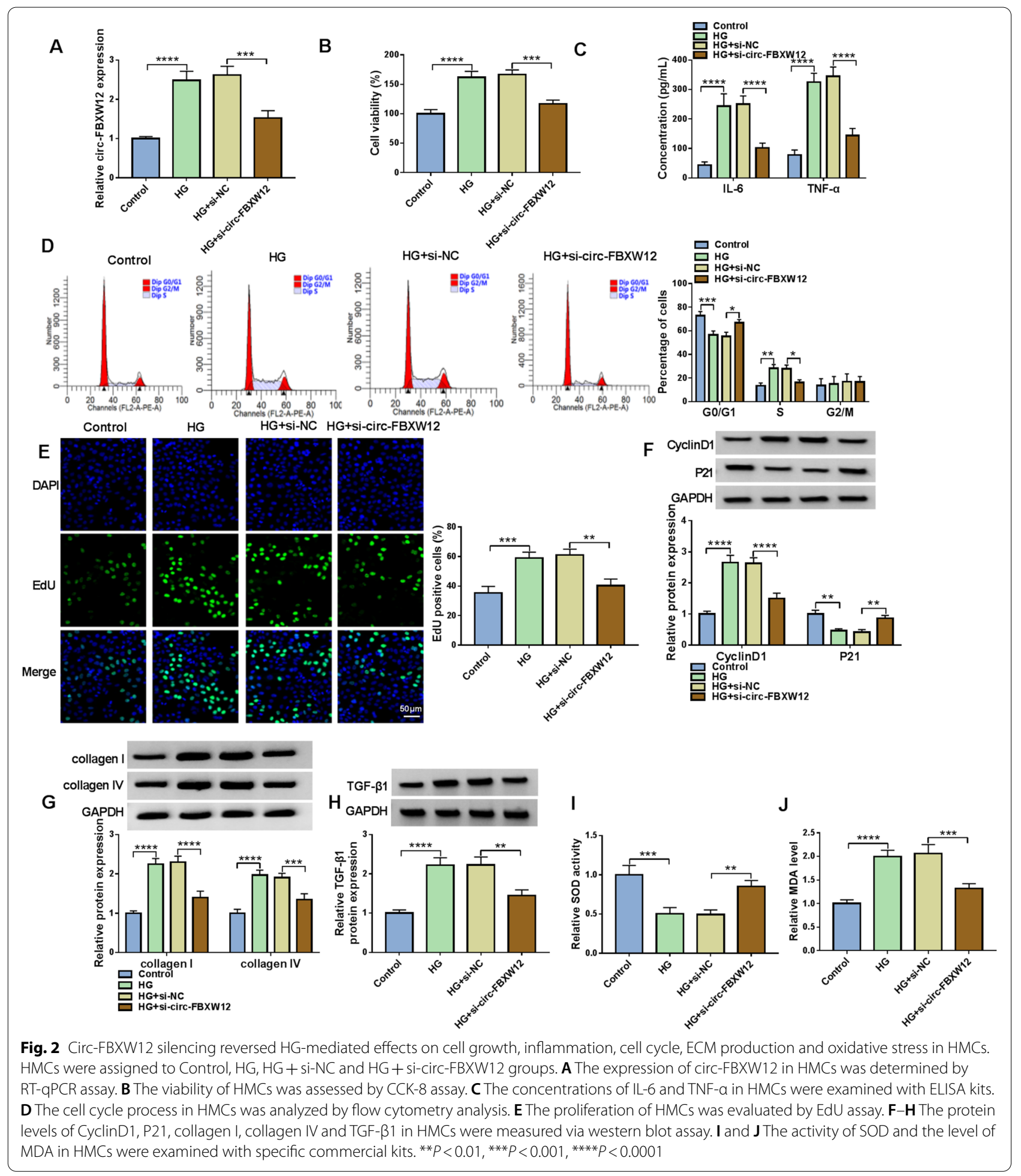

correlation coefficient analysis, there was an inverse correlation between the levels of miR-31-5p and circ-FBXW12 in the serums of DN patients (Fig. 3G). Thereafter, we found that HG-induced circ-FBXW12 upregulation was further promoted by the transfection of circ-FBXW12 in HMCs, indicating that circ-FBXW12 was successfully transfected into HMCs (Fig. 3H). Furthermore, our results presented that circ-FBXW12 knockdown increased miR31-5p expression in HMCs, while circ-FBXW12 overexpression decreased miR-31-5p expression in HMCs 


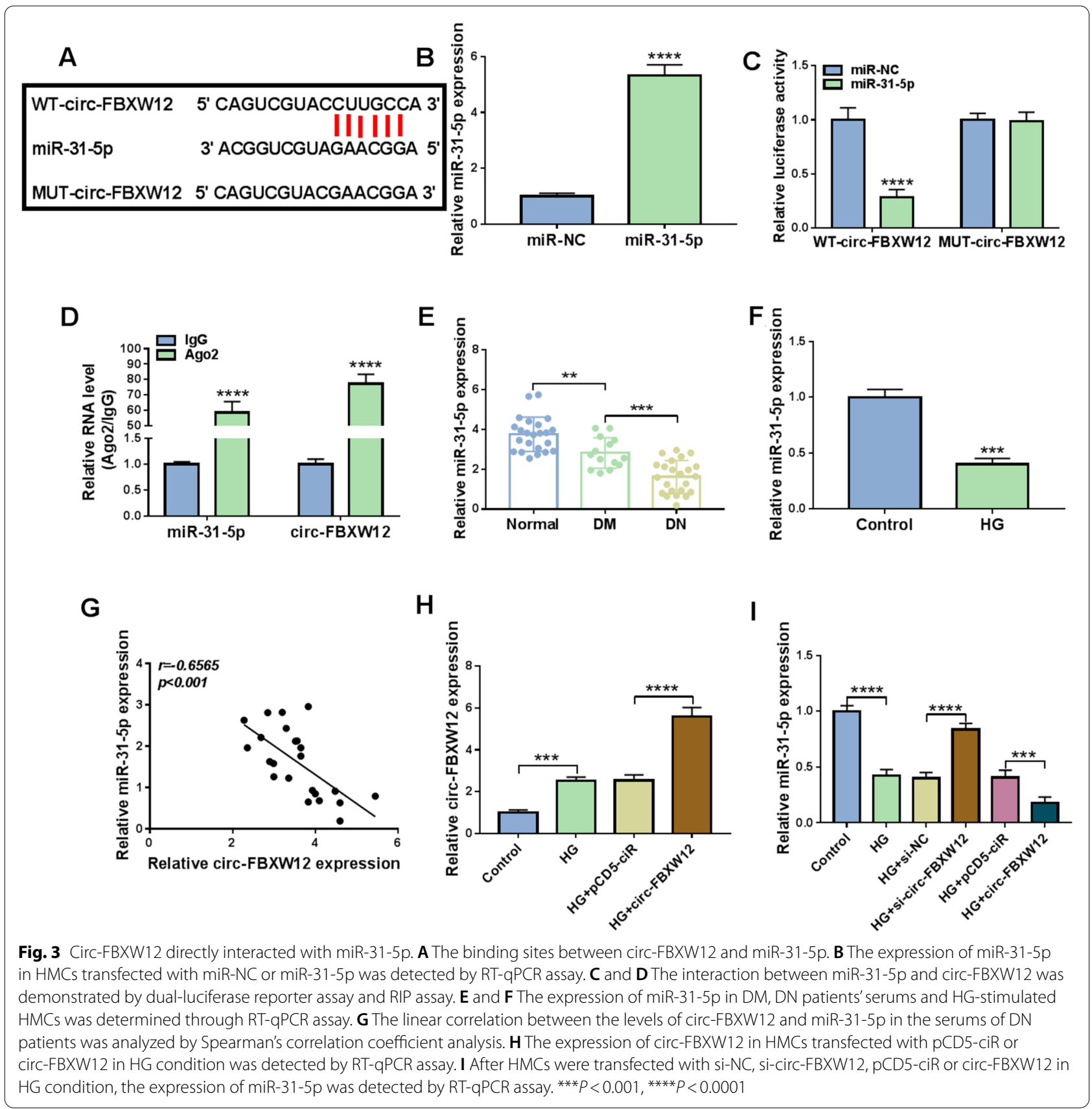

(Fig. 3I). Collectively, circ-FBXW12 directly targeted miR31-5p to negatively alter miR-31-5p expression in HMCs.

\section{Inhibition of miR-31-5p rescued the impacts}

of circ-FBXW12 knockdown on cell growth, inflammation, cell cycle, ECM production and oxidative stress in HG-treated HMCs

As we observed in Fig. 4A, anti-miR-31-5p transfection reduced the expression of miR-31-5p in HMCs compared to anti-miR-NC control groups. Next, we explored the relationship of circ-FBXW12 and miR-31-5p in the progression of HG-stimulated HMCs by transfecting si-NC, si-circ-FBXW12, si-circ-FBXW12+ anti-miR-NC or si-circ-FBXW12+anti-miR-31-5p. It was found that circ-FBXW12 knockdown increased the expression of miR-31-5p in HMCs, while anti-miR-31-5p transfection abolished the effect (Fig. 4B). CCK-8 assay suggested that circ-FBXW12 silencing repressed HG-stimulated HMC viability, with miR-31-5p inhibition reversed the impact (Fig. 4C). ELISA showed that circ-FBXW12 knockdown 


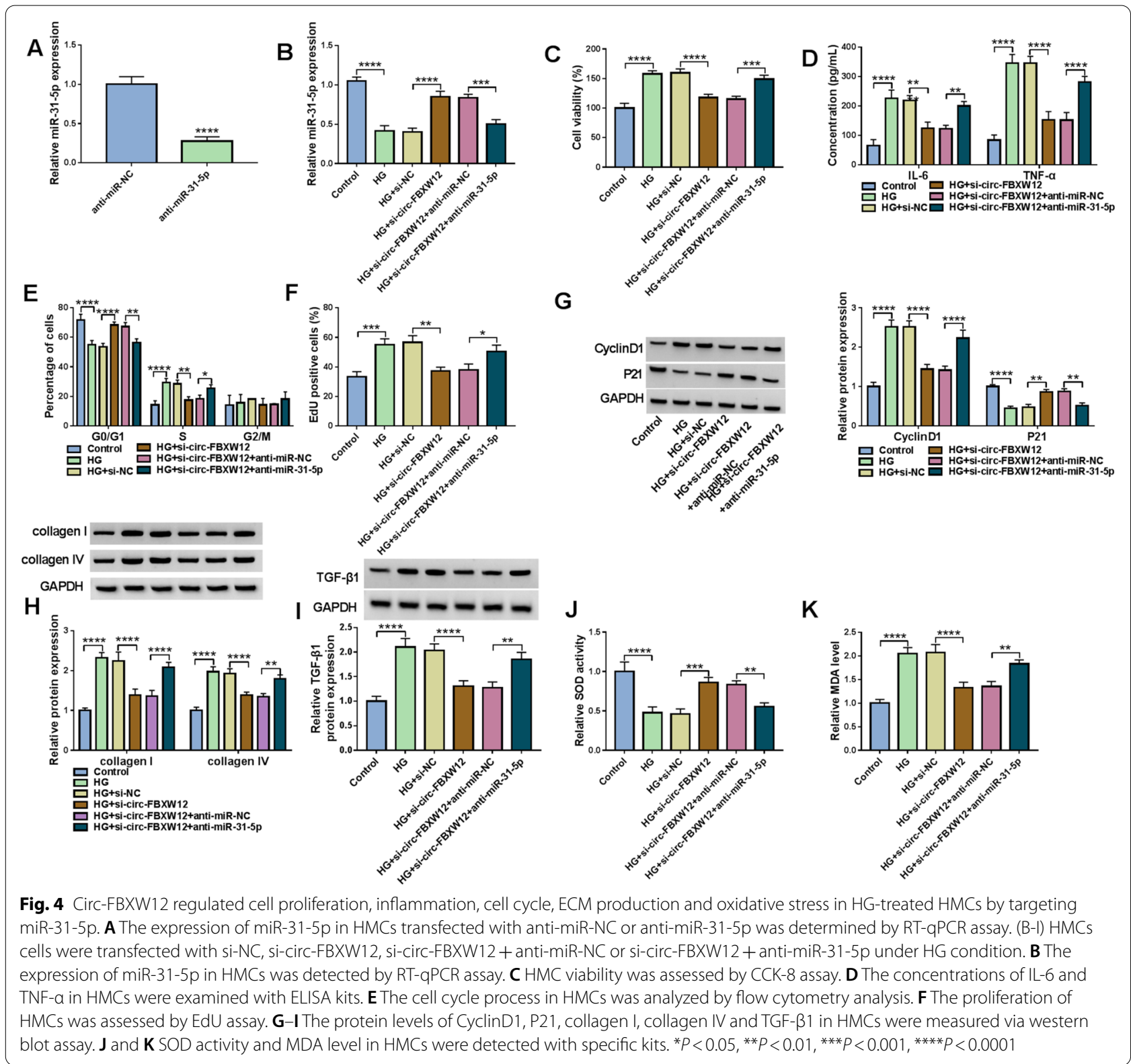

reduced the concentrations of IL- 6 and TNF- $\alpha$ in HGtriggered HMCs, while the effects were weakened by decreasing miR-31-5p (Fig. 4D). As illustrated by flow cytometry analysis, circ-FBXW12 deficiency arrested cell cycle in G0/G1 phase in HG-treated HMCs, whereas miR-31-5p downregulation ameliorated the effect (Fig. 4E). EdU assay indicated the suppressive role of circ-FBXW12 knockdown in HG-treated HMC proliferation was reversed by reducing miR-31-5p (Fig. 4F). We also found that the effects of circ-FBXW12 knockdown on CyclinD1, P21, collagen I, collagen IV and TGF- $\beta 1$ protein levels in HG-treated HMCs were abrogated by miR-31-5p inhibition (Fig. 4G-I). Besides, circ-FBXW12 silencing increased SOD activity and decreased MDA level in HG-treated HMCs, with miR-31-5p reduction ameliorated the impacts (Fig. 4J and K). To summarize, circ-FBXW12 deficiency repressed cell proliferation, inflammation, cell cycle, ECM production and oxidative stress in HG-treated HMCs by sponging miR-31-5p.

\section{LIN28B was the target gene of miR-31-5p}

To further explore the regulatory mechanism of circFBXW12/miR-31-5p in HG-induced HMCs progression, we analyzed starBase v2.0 (http://starbase.sysu. edu.cn/starbase2/) and found that LIN28B might be a target gene of miR-31-5p (Fig. 5A). Then dual-luciferase 
reporter assay and RIP assay were carried out to demonstrate the prediction. The results of dual-luciferase reporter assay showed that the luciferase activity of WT-LIN28B 3'UTR in HMCs was reduced following miR-31-5p overexpression, while the luciferase activity of MUT-LIN28B 3'UTR was not changed (Fig. 5B). RIP assay indicated that miR-31-5p and LIN28B levels were all enhanced in Ago2 immunoprecipitation complexes compared to IgG control groups (Fig. 5C). Moreover, LIN28B mRNA level in DN patients' serum was evidently increased and negatively correlated with miR-31-5p level (Fig. 5D and E). Compared to control groups, LIN28B protein level was increased in HG-stimulated HMCs (Fig. 5F). Besides, miR-31-5p overexpression reduced LIN28B protein level and miR31-5p knockdown elevated LIN28B protein level in
HG-treated HMCs (Fig. 5G). These findings illustrated that miR-31-5p negatively regulated LIN28B expression by direct interaction.

\section{LIN28B knockdown suppressed cell proliferation, inflammation, cell cycle process, ECM production and oxidative stress in HG-treated HMCs}

Subsequently, the functional roles of LIN28B in HGinduced HMCs progression were investigated. As shown in Fig. 6A, si-LIN28B transfection led to a distinct reduction in LIN28B protein level in HG-treated HMCs. CCK-8 assay showed that LIN28B silencing suppressed HG-induced HMC cell viability compared to si-con control groups (Fig. 6B). HG-induced elevation of IL- 6 and TNF- $\alpha$ in HMCs was reversed by silencing LIN28B (Fig. 6C). Flow cytometry analysis showed that the promotional effect of HG treatment

A

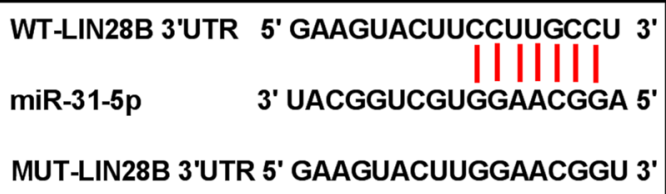

B
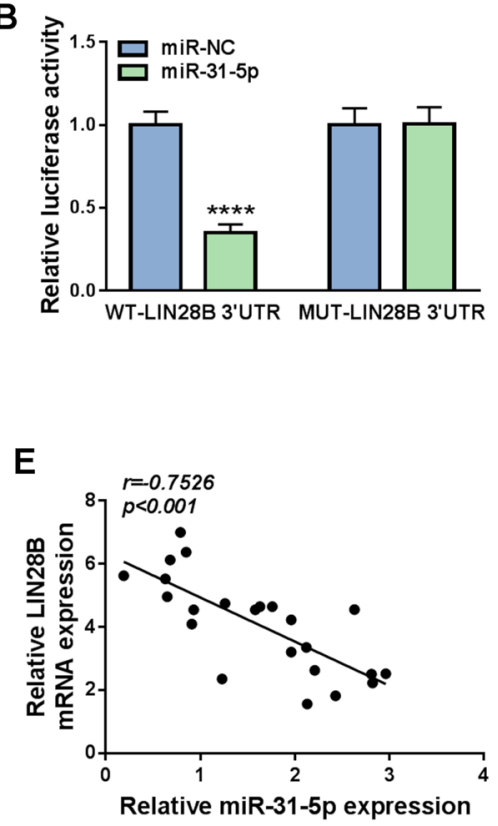

C
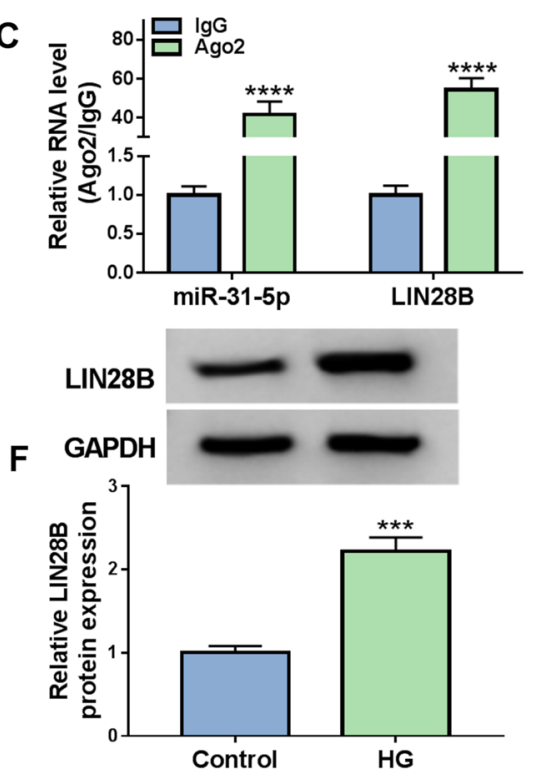

D
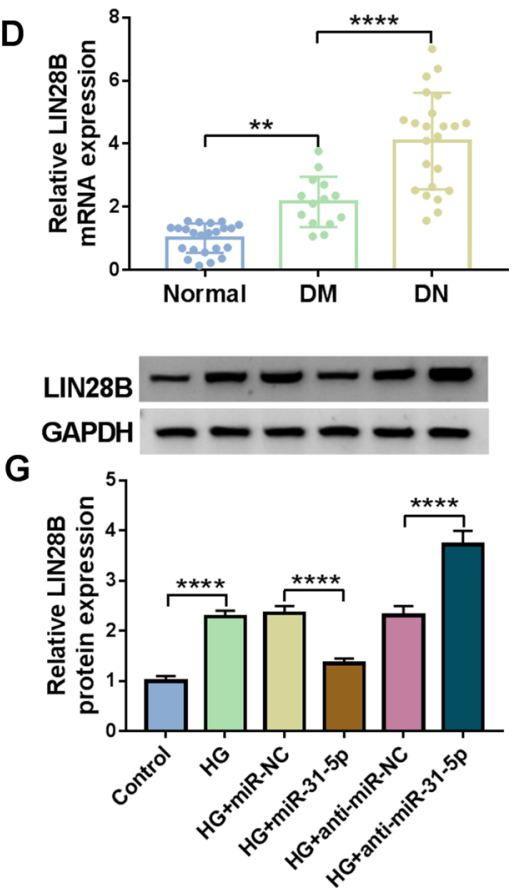

Fig. 5 MiR-31-5p directly targeted LIN28B. A LIN28B contained miR-31-5p binding sites. B and C Dual-luciferase reporter assay and RIP assay were conducted to analyze the interaction between miR-31-5p and LIN28B. D The mRNA level of LIN28B in the serums of DM, DN patients and healthy volunteers was detected by RT-qPCR assay. E The correlation between the levels of mIR-31-5p and LIN28B in DN patients'serums was analyzed by Spearman's correlation coefficient analysis. F The protein level of LIN28B in HG-treated HMCs was measured via western blot assay. G The protein level in HG-treated HMCs transfected with miR-NC, miR-31-5p, anti-miR-NC or anti-miR-31-5p was measured through western blot assay. ${ }^{* * *} P<0.001,{ }^{* * *} P<0.0001$ 


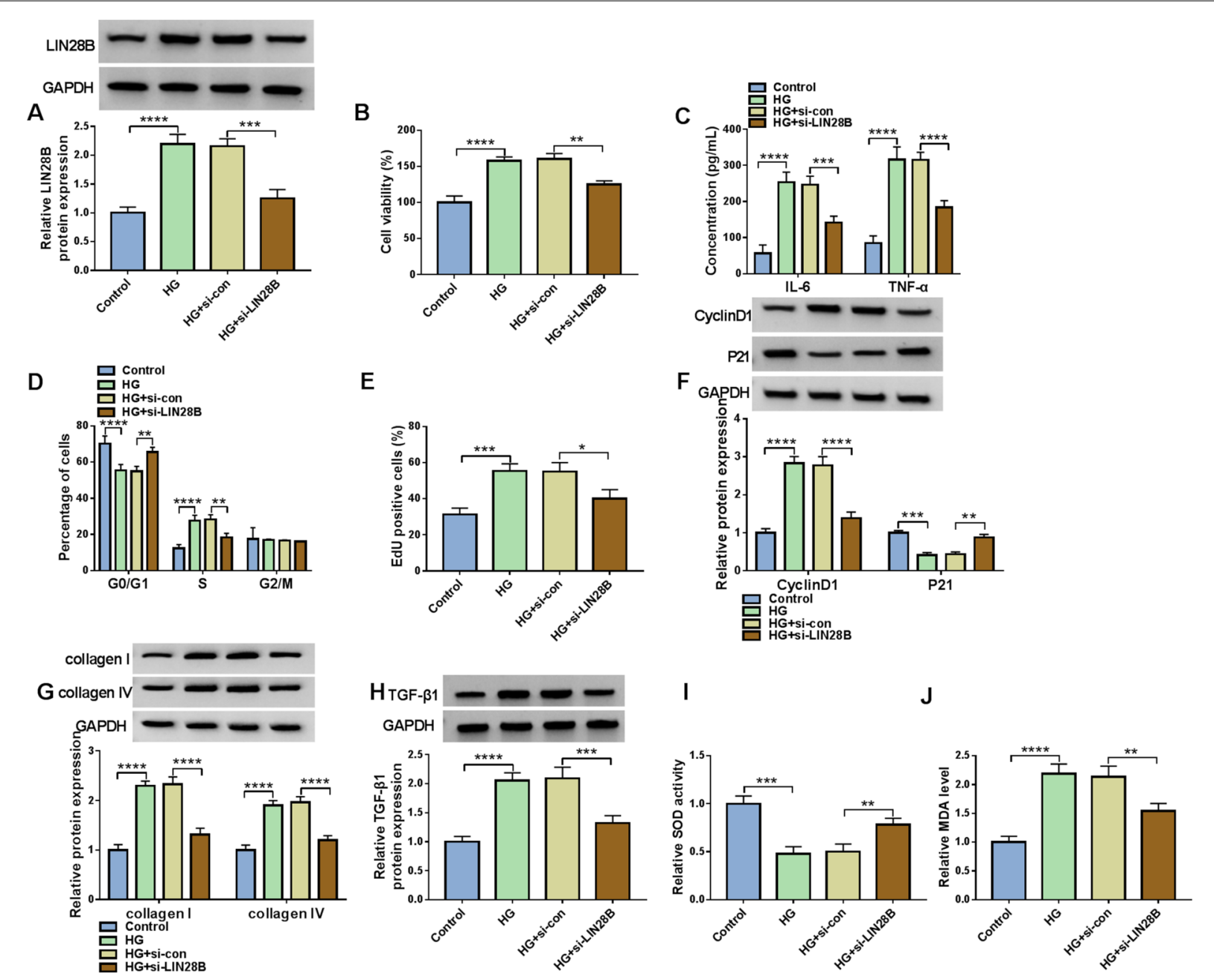

Fig. 6 LIN28B knockdown suppressed cell viability, inflammation, cell cycle process, ECM production and oxidative stress in HG-treated HMCs. HMCs were transfected with si-con or si-LIN28B under HG condition. A The protein level of LIN28B in HMCs was measured via western blot assay. B The viability of HMCs was evaluated by CCK-8 assay. C The levels of IL- 6 and TNF-a in HMCs were determined by ELISA kits. D The cell cycle process in HMCs was analyzed by flow cytometry analysis. E The proliferation of HMCs was tested by EdU assay. F-H The protein levels of CyclinD1, P21, collagen I, collagen IV and TGF- $\beta 1$ in HMCs were measured via western blot assay. I and $\mathbf{J}$ The activity of SOD and the level of MDA in HMCs were measured by relevant kits. ${ }^{*} P<0.05,{ }^{* *} P<0.01,{ }^{* * *} P<0.001,{ }^{* * * *} P<0.0001$

on cell cycle process in HMCs was abated by LIN28B knockdown (Fig. 6D). LIN28B silencing inhibited the ability of HG-treated HMCs to proliferate compared to si-con groups (Fig. 6E). Moreover, LIN28B interference reduced the protein levels of CyclinD1, collagen I, collagen IV and TGF- $\beta 1$ and elevated the protein level of P21 in HG-triggered HMCs (Fig. 6F-H). In addition, we found that LIN28B knockdown enhanced SOD activity and reduced MDA level in HG-stimulated HMCs in comparison with si-con groups (Fig. 6I and J). Collectively, LIN28B knockdown suppressed HGinduced HMCs development.
MiR-31-5p overexpression repressed cell proliferation, inflammation, cell cycle, ECM production and oxidative stress in HG-stimulated HMCs by targeting LIN28B

The transfection of LIN28B overexpression vector elevated LIN28B protein level in HMCs compared to pcDNA control groups (Fig. 7A). MiR-31-5p transfection reduced LIN28B protein level in HG-treated HMCs, while LIN28B transfection reversed the effect (Fig. 7B). CCK-8 assay presented that miR-31-5p overexpression restrained the viability of HG-treated HMCs, while LLIN28B elevation rescued the effect (Fig. 7C). Overexpression of miR-31-5p caused a 


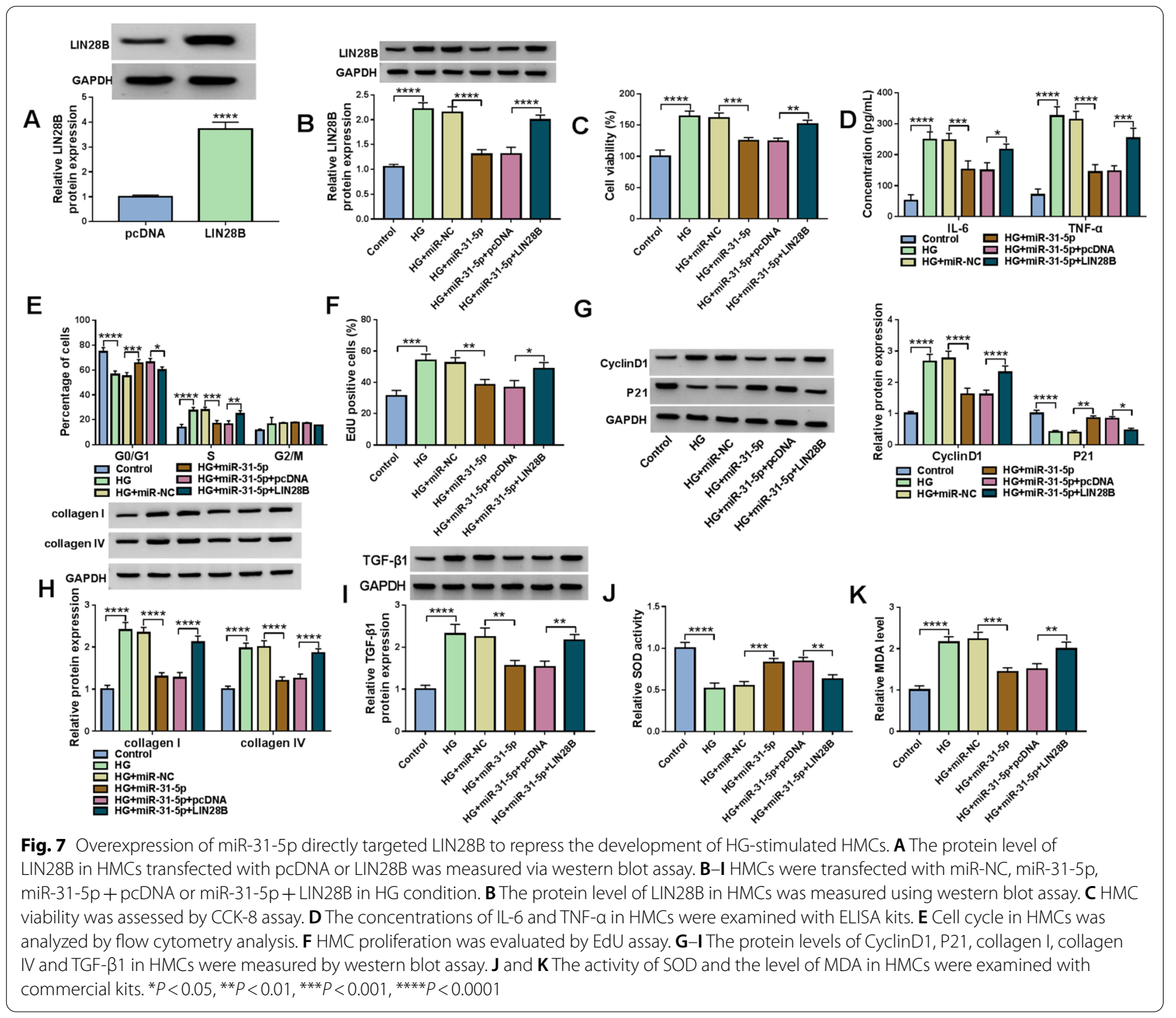

distinct reduction in IL- 6 and TNF- $\alpha$ concentrations in HG-treated HMCs, whereas the impacts were abolished by increasing LIN28B (Fig. 7D). As demonstrated by flow cytometry analysis and EdU assay, miR-31-5p overexpression arrested cell cycle and suppressed cell proliferation in HG-stimulated HMCs, while LIN28B upregulation overturned the impacts (Fig. 7E and F). The protein levels of CyclinD1, collagen I, collagen IV and TGF- $\beta 1$ were reduced and the protein level of P21 was increased in HG-triggered HMCs after miR-31-5p transfection, while LIN28B overexpression weakened the effects (Fig. 7G-I). Additionally, miR-31-5p overexpression enhanced SOD activity and declined MDA level in HG-treated HMCs, with LIN28B elevation ameliorated the effects (Fig. 7J and K). All these findings suggested that miR-31-5p overexpression suppressed the progression of HG-treated HMCs by targeting LIN28B.

\section{Circ-FBXW12 knockdown suppressed LIN28B expression by sponging miR-31-5p}

At last, the relationships of circ-FBXW12, miR-31-5p and LIN28B were analyzed. It was found that circ-FBXW12 silencing resulted in a marked reduction in LIN28B mRNA and protein levels in HG-treated HMCs, while these effects were rescued by the inhibition of miR-31-5p (Fig. 8A and B). 
A

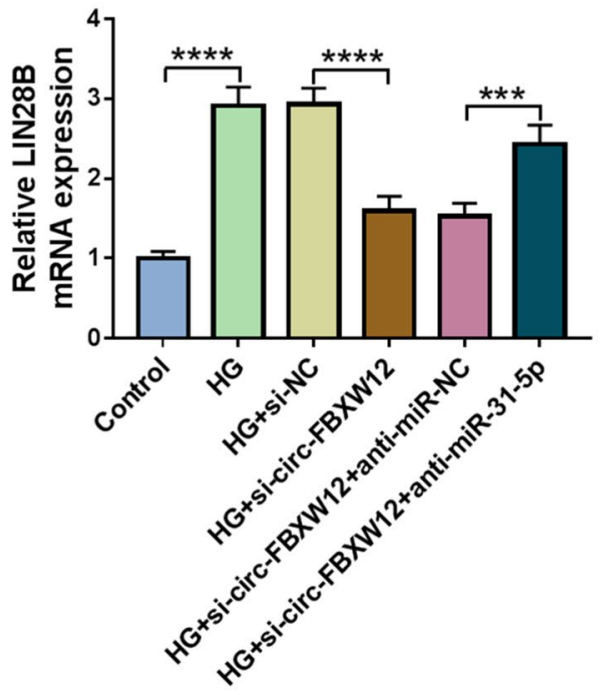

B
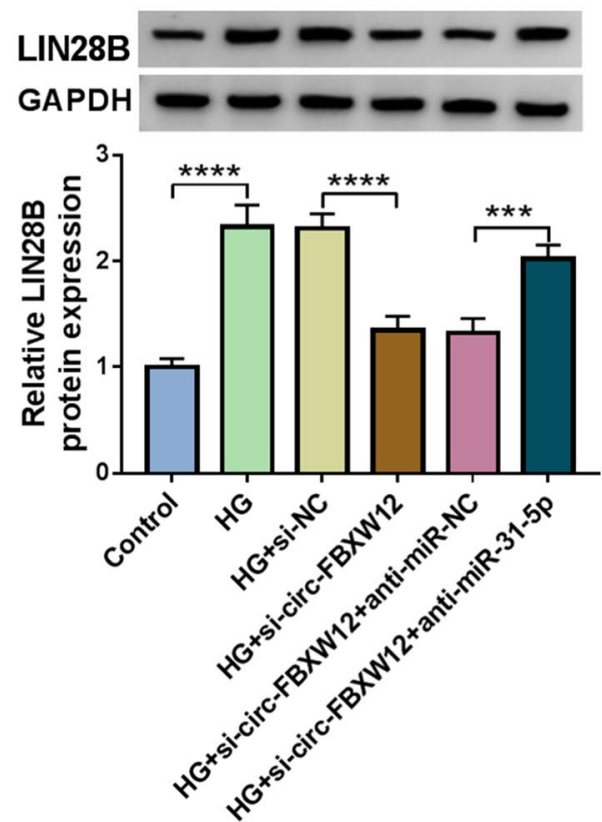

Fig. 8 Circ-FBXW12 regulated LIN28B expression by targeting miR-31-5p. A and B After HMCs were transfected with si-NC, si-circ-FBXW12, si-circ-FBXW12 + anti-miR-NC or si-circ-FBXW12 + anti-miR-31-5p in HG condition, the mRNA and protein levels of LIN28B were determined by RT-qPCR assay and western blot assay, respectively. ${ }^{* * *} P<0.001,{ }^{* * *} P<0.0001$

\section{Discussion}

In the last decade, circRNAs have been gradually identified and discovered to play crucial regulatory roles in a wide range of human diseases [23]. Moreover, the involvement of circRNAs in DN was reported. For example, circ_0000491 level was elevated in DN mice and HG-induced MMCs and promoted ECM production through the mediation of miR-101b and TGF $\beta R I$ [24]. Circ_0037128 silencing repressed MC growth and fibrosis by reducing AKT Serine/Threonine Kinase 3 (AKT3) through adsorbing miR-17-3p [25]. Circ_WBSCR17 contributed to HG-induced HK2 cell inflammation and fibrosis by miR-185-5p/SRY-box transcription factor 6 (SOX6) axis [26].

In this study, circ-FBXW12 level was abnormally increased in DN patients' serums. HG treatment also activated circ-FBXW12 expression in HMCs. Thus, we speculated that circ-FBXW12 might exert a function in DN. Proliferation, inflammation, and fibrosis of MCs are important pathophysiological features of $\mathrm{DN}$ [27, 28]. Herein, our results showed that HG promoted cell proliferation and inflammatory factors release in HMCs, while circ-FBXW12 knockdown reversed the impacts. In addition, the enhancement of oxidative stress caused by hyperglycemia can aggravate DN progression [29]. In this work, HG-triggered oxidative injury in HMCs was abolished by decreasing circ-FBXW12. As DN is a metabolic disorder characterized by ECM accumulation, which can be induced by some pathological conditions such as oxidative stress and autophagy [30,31]. Thus, we explored the effect of circ-FBXW12 on ECM by detecting the ECM related markers, including collagen I, collagen IV and TGF- $\beta 1[32,33]$. Our results demonstrated that circ-FBXW12 knockdown reduced the levels of collagen I, collagen IV and TGF- $\beta 1$ in HG-triggered HMCs, suggesting the suppression of ECM production. All these findings indicated that circ-FBXW12 knockdown was able to alleviate HG-stimulated HMC damage.

Thereafter, the underlying mechanism of circ-FBXW12 was investigated. As a result, we demonstrated that circ-FBXW12 served as the sponge for miR-31-5p and miR-31-5p was lowly expressed in DN patients and HGtriggered HMCs. Though miR-31-5p was reported to be targeted by several circRNAs, such as circ_0035483 [34], circ_0063517 [35] and circ-BPTF [36], the relationship between circ-FBXW12 and miR-31-5p was firstly clarified. Moreover, miR-31-5p inhibition abated circFBXW12 knockdown-mediated impact on HG-induced HMC cell injury, indicating that circ-FBXW12 altered HG-induced HMC damage by decoying miR-31-5p. Additionally, our results explored the exact functions of miR-31-5p in HG-induced HMCs. The outcomes exhibited that miR-31-5p overexpression restrained cell proliferation, inflammation, ECM enrichment and oxidative in HG-stimulated HMCs. 
Furthermore, it was demonstrated that miR-31-5p directly targeted LIN28B and circ-FBXW12 positively regulated LIN28B expression by sponging miR-31-5p in HMCs. LIN28B silencing ameliorated HG-driven cell growth, inflammatory response, ECM accumulation and oxidative impairment in HMCs. A previous study reported that miR-379-5p could hamper MMC proliferation and ECM production by targeting LIN28B [37]. In this research, LIN28B elevation relieved the influence of miR-31-5p overexpression on HG-induced injury of HMCs.

In conclusion, we elaborated the importance of circFBXW12 in HG-induced HMCs. We demonstrated that circ-FBXW12 knockdown could reverse HG-induced HMC cell injury by altering cell growth, inflammation, ECM enrichment and oxidative stress through miR31-5p/LIN28B axis. Our findings provided clues for understanding the significance of circRNAs in DN and contributed to develop better diagnostic and therapeutic strategies.

However, there are some limitations in our study. For example, the number of patients was limited. Moreover, we did not verify our results in other DN cell model and DN mice model.

\begin{abstract}
Abbreviations
DN: Diabetic nephropathy; CircRNAs: Circular RNAs; FBXW12: F-box/WD repeat-containing protein 12; RT-qPCR: Reverse transcription quantitative polymerase chain reaction; LIN28B: Lin-28 homolog B; CCK-8: Cell counting kit-8; EdU: 5-Ethynyl-2'- deoxyuridine; ELISA: Enzyme linked immunosorbent assay; SOD: Superoxide dismutase; MDA: Malondialdehyde; RIP: RNA immunoprecipitation; HG: High glucose; HMCs: Human mesangial cells; ECM: Extracellular matrix; MC: Mesangial cell; ncRNAs: Non-coding RNAs; miRNA: MicroRNA; MMCs: Mouse mesangial cells; MAPK6: Mitogen-activated protein kinase 6; Bach1: BTB Domain And CNC Homolog 1; CCL19: C-C motif chemokine ligand 19; Sema3A: Semaphorin 3A; DMEM: Dulbecco's modified Eagle's medium; FBS: Fetal bovine serum; siRNA: Small interfering RNA; GAPDH: Glyceraldehyde 3-phosphate dehydrogenase; IL-6: Interleukin-6; TNF-a: Tumour necrosis factor a; PBS: Phosphate buffer saline; PI: Propidium iodide; RIPA: Radioimmunoprecipitation assay; SDS-PAGE: Sodium dodecyl sulfonate-polyacrylamide gel; PVDF: Polyvinylidene difluoride membranes; ECL: Enhanced chemiluminescence; AKT3: AKT serine/threonine kinase 3; SOX6: SRY-box transcription factor 6.
\end{abstract}

\section{Acknowledgements}

None.

\section{Authors' contributions}

AS participated in the design of the work, methodology, data interpretation, and analysis for the work, carried out the statistical analysis and drafted the manuscript. NS participated in the methodology, data interpretation, and analysis for the work. XL and ZH designed the study, participated in data interpretation and methodology. All authors read and approved the final manuscript.

\section{Funding}

There is no funding to report.

Availability of data and materials

Data analyzed for this study will be available on a reasonable request.

\section{Declarations}

Ethics approval and consent to participate

The research related to human sample use has been complied with all the relevant national regulations, were acquired after the research was approved by the Ethics Committee of Zibo First Hospital.

\section{Consent for publication}

Written informed consents were provided by the participants.

\section{Competing interests}

The authors declare that they have no competing interest.

\section{Author details}

${ }^{1}$ Department of Endocrinology, Zibo First Hospital, Zibo 255200, Shandong, China. ${ }^{2}$ Chinese Traditional College of Changchun University of Chinese Medicine, Changchun 130022, Jilin, China. ${ }^{3}$ Department of Thoracic Surgery, Zibo Central Hospital, Zibo 255000, Shandong, People's Republic of China.

${ }^{4}$ Department of Pathology, Zibo Central Hospital, No. 54 Gongqingtuan West Road, Zhangdian District, Zibo 255000, Shandong, People's Republic of China.

Received: 29 March 2021 Accepted: 12 November 2021

Published online: 04 December 2021

\section{References}

1. Zimmet P, Alberti KG, Magliano DJ, Bennett PH. Diabetes mellitus statistics on prevalence and mortality: facts and fallacies. Nat Rev Endocrinol. 2016;12(10):616-22.

2. Barutta F, Bruno G, Grimaldi S, Gruden G. Inflammation in diabetic nephropathy: moving toward clinical biomarkers and targets for treatment. Endocrine. 2015;48(3):730-42.

3. Letelier CEM, Ojeda CASM, Provoste JJR, Zaror CJF. Pathophysiology of diabetic nephropathy: a literature review. Medwave. 2017:17(1): e6839.

4. Lu Z, Liu N, Wang F. Epigenetic regulations in diabetic nephropathy. J Diabetes Res. 2017;2017:7805058.

5. Brownlee M. The pathobiology of diabetic complications: a unifying mechanism. Diabetes. 2005;54(6):1615-25.

6. Xu K, Guo L, Bu H, Wang H. Daphnetin inhibits high glucose-induced extracellular matrix accumulation, oxidative stress and inflammation in human glomerular mesangial cells. J Pharmacol Sci. 2019;139(2):91-7.

7. Chen LL. The biogenesis and emerging roles of circular RNAs. Nat Rev Mol Cell Biol. 2016:17(4):205-11.

8. Rong D, Sun H, Li Z, Liu S, Dong C, Fu K, Tang W, Cao H. An emerging function of circRNA-miRNAs-mRNA axis in human diseases. Oncotarget. 2017;8(42):73271-81

9. Liu H, Wang X, Wang ZY, Li L. Circ_0080425 inhibits cell proliferation and fibrosis in diabetic nephropathy via sponging miR-24-3p and targeting fibroblast growth factor 11. J Cell Physiol. 2020;235(5):4520-9.

10. Yao T, Zha D, Hu C, Wu X. Circ_0000285 promotes podocyte injury through sponging miR-654-3p and activating MAPK6 in diabetic nephropathy. Gene. 2020;747: 144661.

11. Wang Y, Qi Y, Ji T, Tang B, Li X, Zheng P, Bai S. Circ_LARP4 regulates high glucose-induced cell proliferation, apoptosis, and fibrosis in mouse mesangial cells. Gene. 2021;765: 145114

12. Wang W, Feng J, Zhou H, Li Q. Circ_0123996 promotes cell proliferation and fibrosisin mouse mesangial cells through sponging miR-149-5p and inducing Bach1 expression. Gene. 2020;761: 144971.

13. Kato M, Natarajan R. MicroRNAs in diabetic nephropathy: functions, biomarkers, and therapeutic targets. Ann N Y Acad Sci. 2015;1353:72-88.

14. Sun J, Wang J, Lu W, Xie L, Lv J, Li H, Yang S. MiR-325-3p inhibits renal inflammation and fibrosis by targeting CCL19 in diabetic nephropathy. Clin Exp Pharmacol Physiol. 2020:47(11):1850-60.

15. Fu Y, Wang C, Zhang D, Chu X, Zhang Y, Li J. miR-15b-5p ameliorated high glucose-induced podocyte injury through repressing apoptosis, oxidative stress, and inflammatory responses by targeting Sema3A. J Cell Physiol. 2019;234(11):20869-78. 
16. Rovira-Llopis S, Escribano-Lopez I, Diaz-Morales N, lannantuoni F, LopezDomenech S, Andujar I, Jover A, Pantoja J, Pallardo LM, Banuls C, et al. Downregulation of miR-31 in diabetic nephropathy and its relationship with inflammation. Cell Physiol Biochem. 2018;50(3):1005-14.

17. Zhang J, Hu K, Yang YQ, Wang Y, Zheng YF, Jin Y, Li P, Cheng L. LIN28B-AS1IGF2BP1 binding promotes hepatocellular carcinoma cell progression. Cell Death Dis. 2020;1 1(9):741.

18. Lovnicki J, Gan Y, Feng T, Li Y, Xie N, Ho CH, Lee AR, Chen X, Nappi L, Han $\mathrm{B}$, et al. LIN28B promotes the development of neuroendocrine prostate cancer. J Clin Invest. 2020;130(10):5338-48.

19. Tao T, Shi H, Mariani L, Abraham BJ, Durbin AD, Zimmerman MW, Powers JT, Missios P, Ross KN, Perez-Atayde AR, et al. LIN28B regulates transcription and potentiates MYCN-induced neuroblastoma through binding to ZNF143 at target gene promotors. Proc Natl Acad Sci USA. 2020;117(28):16516-26.

20. Keskin T, Bakaric A, Waszyk P, Boulay G, Torsello M, Cornaz-Buros S, Chevalier N, Geiser T, Martin P, Volorio A, et al. LIN28B underlies the pathogenesis of a subclass of Ewing sarcoma LIN28B control of EWS-FLI1 stability. Cell Rep. 2020;30(13):4567-4583 e4565.

21. Park JT, Kato M, Lanting L, Castro N, Nam BY, Wang M, Kang SW, Natarajan R. Repression of let-7 by transforming growth factor-beta 1-induced Lin28 upregulates collagen expression in glomerular mesangial cells under diabetic conditions. Am J Physiol Renal Physiol. 2014;307(12):F1390-1403.

22. Gwon MG, An HJ, Gu H, Kim YA, Han SM, Park KK. Apamin inhibits renal fibrosis via suppressing TGF-beta1 and STAT3 signaling in vivo and in vitro. J Mol Med. 2021. https://doi.org/10.1007/s00109-021-02087-x.

23. Chen I, Chen CY, Chuang TJ. Biogenesis, identification, and function of exonic circular RNAs. Wiley Interdiscip Rev RNA. 2015;6(5):563-79.

24. Mou X, Chenv JW, Zhou DY, Liu K, Chen L, Zhou D, Hu YB. A novel identified circular RNA, circ_0000491, aggravates the extracellular matrix of diabetic nephropathy glomerular mesangial cells through suppressing miR101b by targeting TGFbetaRI. Mol Med Rep. 2020;22(5):3785-94.

25. Wang Q, Cang Z, Shen L, Peng W, Xi L, Jiang X, Ge X, Xu B, Huang S. circ_0037128/miR-17-3p/AKT3 axis promotes the development of diabetic nephropathy. Gene. 2021;765: 145076.

26. Li G, Qin Y, Qin S, Zhou X, Zhao W, Zhang D. Circ_WBSCR17 aggravates inflammatory responses and fibrosis by targeting miR-185-5p/SOX6 regulatory axis in high glucose-induced human kidney tubular cells. Life Sci. 2020;259: 118269
27. Tung CW, Hsu YC, Shih YH, Chang PJ, Lin CL. Glomerular mesangial cell and podocyte injuries in diabetic nephropathy. Nephrology. 2018;23(Suppl 4):32-7.

28. Mora C, Navarro JF. Inflammation and diabetic nephropathy. Curr Diab Rep. 2006;6(6):463-8.

29. Zhu X, Shi J, Li H. Liquiritigenin attenuates high glucose-induced mesangial matrix accumulation, oxidative stress, and inflammation by suppression of the NF-kappaB and NLRP3 inflammasome pathways. Biomed Pharmacother. 2018;106:976-82.

30. Liu HF, Liu H, Lv LL, Ma KL, Wen Y, Chen L, Liu BC. CCN3 suppresses TGFbeta1-induced extracellular matrix accumulation in human mesangial cells in vitro. Acta Pharmacol Sin. 2018;39(2):222-9.

31. Shemesh II, Rozen-Zvi B, Kalechman Y, Gafter U, Sredni B. AS101 prevents diabetic nephropathy progression and mesangial cell dysfunction: regulation of the AKT downstream pathway. PLoS ONE. 2014;9(12): e114287.

32. Schena FP, Gesualdo L. Pathogenetic mechanisms of diabetic nephropathy. J Am Soc Nephrol. 2005;16(Suppl 1):S30-33.

33. Tang PM, Zhang YY, MakTS, Tang PC, Huang XR, Lan HY. Transforming growth factor-beta signalling in renal fibrosis: from Smads to non-coding RNAs. J Physiol. 2018:596(16):3493-503.

34. Liu Z, Wang R, Zhu G. Circ_0035483 functions as a tumor promoter in renal cell carcinoma via the miR-31-5p-mediated HMGA1 upregulation. Cancer Manag Res. 2021;13:693-706.

35. Li W, Yu N, Fan L, Chen SH, Wu JL. Circ_0063517 acts as ceRNA, targeting the miR-31-5p-ETBR axis to regulate angiogenesis of vascular endothelial cells in preeclampsia. Life Sci. 2020;244: 117306.

36. Bi J, Liu H, Cai Z, Dong W, Jiang N, Yang M, Huang J, Lin T. Circ-BPTF promotes bladder cancer progression and recurrence through the miR31-5p/RAB27A axis. Aging. 2018;10(8):1964-76.

37. Li N, Wang LJ, Xu WL, Liu S, Yu JY. MicroRNA3795p suppresses renal fibrosis by regulating the LIN28/let7 axis in diabetic nephropathy. Int J Mol Med. 2019;44(5):1619-28.

\section{Publisher's Note}

Springer Nature remains neutral with regard to jurisdictional claims in published maps and institutional affiliations.
Ready to submit your research? Choose BMC and benefit from:

- fast, convenient online submission

- thorough peer review by experienced researchers in your field

- rapid publication on acceptance

- support for research data, including large and complex data types

- gold Open Access which fosters wider collaboration and increased citations

- maximum visibility for your research: over $100 \mathrm{M}$ website views per year

At BMC, research is always in progress.

Learn more biomedcentral.com/submissions 